\title{
A Numerical Study of Slip System Evolution in Ultra-Thin Stainless Steel Foil
}

\author{
Zhongkai Ren ${ }^{1} \oplus$, Wanwan Fan ${ }^{1}$, Jie Hou ${ }^{2}$ and Tao Wang ${ }^{1, *}$ \\ 1 College of Mechanical and Vehicle Engineering, Taiyuan University of Technology, Taiyuan 030024, China; \\ zhongkai_0808@126.com (Z.R.); tyutfww@163.com (W.F.) \\ 2 Analysis and Test Center, Taiyuan University of Science and Technology, Taiyuan 030024, China; houjie@yeah.net \\ * Correspondence: tyutwt@163.com; Tel.: +86-187-3517-0186
}

Received: 13 May 2019; Accepted: 3 June 2019; Published: 5 June 2019

\begin{abstract}
In order to quantitatively describe the effect of the initial grain orientation on the inhomogeneous deformation of 304 austenitic stainless steel foil during tension, a three-dimensional uniaxial tension model was established, based on the crystal plasticity finite element method (CPFEM) and Voronoi polyhedron theory. A three-dimensional representative volume element (RVE) was used to simulate the slip deformation of 304 stainless steel foil with five typical grain orientations under the same engineering strain. The simulation results show that the number and characteristics of active slip systems and the deformation degree of the grain are different due to the different initial grain orientations. The slip systems preferentially initiate at grain boundaries and cause slip system activity at the interior and free surface of the grain. The Brass, S, and Copper oriented 304 stainless steel foil exhibits a high strain hardening index, which is beneficial to strengthening. However, the Cube and Goss oriented 304 stainless steel foil has a low deformation resistance and is prone to plastic deformation.
\end{abstract}

Keywords: ultra-thin foil; slip system evolution; tensile process; crystal plasticity; numerical simulation; grain orientation

\section{Introduction}

Precision stainless steel foil with thickness ranging from 0.01 to $0.1 \mathrm{~mm}$ is a high-end steel product, which has been widely used in micro-manufacturing, micro-electromechanical, and other sophisticated micro-products [1]. With the increasing miniaturization of components, higher requirements are put forward for high-quality precision stainless steel, such as good ductility, high dimensional accuracy, and low surface roughness [2,3]. When the foil thickness is reduced to microscale, the anisotropy of the material increases, and the size effects become significant [4,5]. In this case, the grain morphology, size, orientation, and grain boundary have important impacts on the plastic deformation behavior $[6,7]$. Therefore, the traditional macroscopic plastic deformation theory appears to be no longer applicable to the plastic deformation analysis of the stainless steel foil in microscale.

The crystal plasticity finite element method (CPFEM) is widely used to homogenize the discrete dislocations and analyze the macroscopic effects of dislocation slip, based on the continuum theory, which reflects the microstructural characteristics of materials [8-11]. The mechanisms involved in the macro and micro-deformation of non-ferrous metal materials during tensile deformation have been studied by many scholars using CPFEM. Si et al. [12] established a polycrystalline model based on CPFEM and Voronoi polyhedron theory, and studied the effect of grain size on inhomogeneous deformation and grain rotation during the deformation process of polycrystalline aluminum. The results showed that the plastic deformation occurred preferentially at grain boundaries, and the multi-system slip caused local hardening near grain boundaries. Hama and Takuda $[13,14]$ used CPFEM to analyze the hardening and unloading behavior of magnesium alloy strips during tensile deformation. They found that the 
pyramidal slip system dominated in the loading process, and only the base slip systems were activated during unloading. Ritz and Dawson [15] studied the effect of grain morphology on tensile deformation of aluminum alloys using CPFEM, and pointed out that grain morphology had little effect on the anisotropy of elastic deformation but significantly affected the micro-stress distribution in the grain interior and at the grain boundary. Pi et al. [16] analyzed the effects of polycrystalline model type and tensile strain rate on deformation, necking, and texture evolution of polycrystalline aluminum during the tensile process by CPFEM. Their results showed that the stress increased with the increase in strain rate, and the calculated results by CPFEM were closer to the experimental results.

In order to balance the relationship between the total central processing unit (CPU) calculation time and the model size, the representative volume element (RVE) model with the smallest number of grains was adopted by many scholars to reflect the mechanical behavior of macroscopic samples. Nakamachi et al. [17] proposed a crystalline homogenization algorithm based on multi-scale asymptotic series expansion, established the RVE model for a micro polycrystal structure that could satisfy the periodicity condition of crystal orientation distribution, and verified the accuracy of the RVE model through analytical and statistical examinations. Jigh et al. [18] applied the RVE model to predict the mechanical behavior of metal foams with real microstructure, and studied the effects of different boundary conditions on mechanical properties. Zheng et al. [19] established the RVE model based on CPFEM to predict the influence of structural parameters and relative density of multi-stage honeycomb on its equivalent elastic parameters. The accuracy of the RVE model was verified by comparing with the elastic parameters calculated by the relevant theoretical analytical formula. Kamiński et al. [20] used the iterative and generalized stochastic perturbation technique as a finite element method to determine the effective elasticity tensor for rubber reinforced with the carbon black particles, and applied this to the homogenization problem of the RVE model in such a composite. This study showed some significant differences between numerical and analytical homogenization methods in the context of geometrical uncertainty in the RVE of the composite.

Based on the above literature reviews, it is clear that grain size and morphology as well as strain rate do have an important effect on the plastic deformation behavior of materials. At present, research on the effect of grain orientation on the tensile deformation of 304 austenitic stainless steels, based on the three-dimensional CPFEM model, is still limited. In the present paper, a three-dimensional RVE uniaxial tension model is established based on the CPFEM and the Voronoi polyhedron theory. The effects of initial grain orientation on the inhomogeneous deformation and evolution of slip systems of 304 stainless steel foil during uniaxial tension are analyzed in detail.

\section{Crystal Plasticity Model}

The main physical mechanism of plastic deformation of the face-centered cubic (FCC) metal at room temperature is dislocation slip [21]. According to the visco-plasticity criterion proposed by Schmid's law [22], the relationship between the shear strain rate $\dot{\gamma}^{\alpha}$ and the resolved shear stress $\tau^{\alpha}$ of each slip system is established in the form of the exponential function as:

$$
\dot{\gamma}^{\alpha}=\dot{\gamma}_{0}^{\alpha} f^{\alpha}\left(\frac{\tau^{\alpha}}{\tau_{c}^{\alpha}}\right)
$$

where the resolved shear stress $\tau^{\alpha}$ is the driving force of the slip motion. The slip system $\alpha$ starts when the resolved shear stress reaches the critical value $\tau_{c}^{\alpha}$.

The material rate sensitivity formula, established by Hutchinson [23], is as follows:

$$
f^{\alpha}=\frac{\tau^{\alpha}}{\tau_{c}^{\alpha}} \times\left|\frac{\tau^{\alpha}}{\tau_{c}^{\alpha}}\right|^{n-1}
$$

where $\dot{\gamma}_{0}{ }^{\alpha}$ is the reference shear strain rate of slip system $\alpha, n$ is the rate sensitive coefficient, $\mathrm{n}=0$ and $\mathrm{n}=\infty$ correspond to viscoelasticity and rate-independent materials, respectively, $\tau^{\alpha}$ is the resolved shear stress of the slip system $\alpha, \tau_{c}^{\alpha}$ represents the dislocation slip resistance or critical resolved shear 
stress of the slip system $\alpha$, which is the accumulation of the shear strain of the corresponding slip system on the hardening of the slip system $\alpha . \tau_{c}^{\alpha}$ is expressed by:

$$
\dot{\tau}_{c}{ }^{\alpha}=\sum_{\beta=1}^{N} h_{\alpha \beta}|\dot{\gamma \beta}|,
$$

where $\gamma^{\beta}$ is the shear strain rate of the slip system $\beta$ and $h_{\alpha \beta}$ is the latent hardening coefficient.

In the present paper, the hardening model proposed by Bassani and $\mathrm{Wu}[24,25]$ was used to describe the interactions between different slip systems with different slip coefficients, which can accurately reflect the slipping process of all three hardening stages of FCC metal. The self-hardening moduli $h_{\alpha \alpha}$ can be expressed as follows:

$$
\begin{gathered}
h_{\alpha \alpha}=\left[\left(h_{0}-h_{s}\right) \operatorname{sech}^{2}\left(\frac{\left(h_{0}-h_{s}\right) \gamma^{\alpha}}{\tau_{s}-\tau_{0}}+h_{s}\right)\right] \times G\left(\gamma^{\beta} ; \beta \neq \alpha\right) \\
G\left(\gamma^{\beta} ; \beta \neq \alpha\right)=1+\sum_{\beta=1 \beta \neq \alpha}^{N} f_{\alpha \beta} \tanh \left(\frac{\gamma^{\beta}}{\gamma_{0}}\right),
\end{gathered}
$$

where $h_{0}$ is the initial hardening modulus; $h_{s}$ is the hardening modulus in the easy slip stage; $\tau_{0}$ is the initial critical resolved shear stress; $\tau_{s}$ is the critical shear stress saturation value; $\gamma_{0}$ is the reference shear strain; and $f_{\alpha \beta}$ is the interaction coefficient between the $\alpha$ and $\beta$ slip system. The factor depends on the geometric relationship of the two slip systems, which is represented by five constants $a_{i}(i=1 \sim 5)$.

The latent hardening moduli $h_{\alpha \beta}$ is given by:

$$
h_{\alpha \beta}=q h_{\alpha \alpha}
$$

where $q$ is the latent hardening parameter.

The above constitutive model was compiled into the user-defined material subroutine (UMAT) of ABAQUS finite element software by Fortran language for subsequent finite element simulation [26], and the combination of crystal plasticity constitutive theory and finite element method was realized. A numerical integration method using implicit differential equations was adopted to solve the rate-dependent crystal plasticity equation. The UMAT used the ABAQUS internal interface to communicate with the main solver. The specific process is shown in Figure 1.

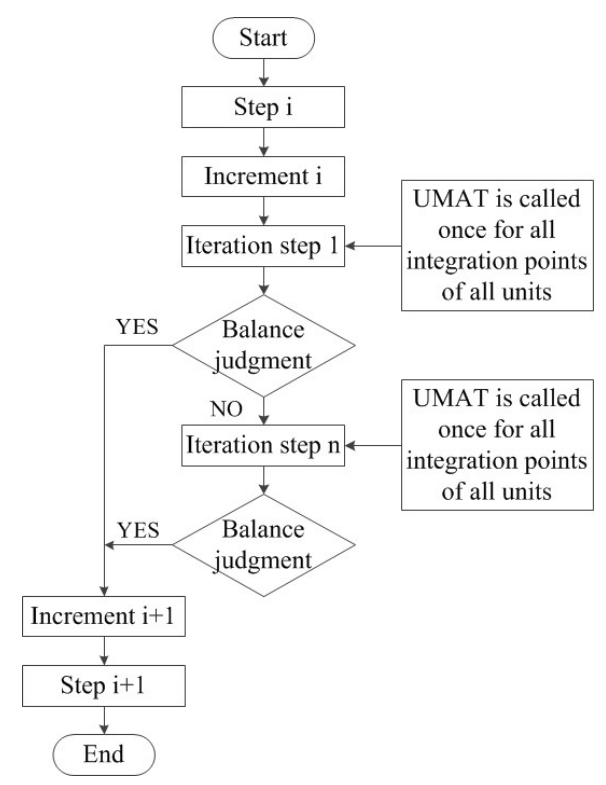

Figure 1. The internal operation flow chart of ABAQUS finite element software. 


\section{Finite Element Model for Stainless Steel Foil}

X-ray diffraction (XRD) phase analysis of the ultra-thin 304 stainless steel foil $(0.05 \mathrm{~mm})$ showed that the main phase constituent was FCC austenite. The elastic constants of single crystal austenite are $\mathrm{C}_{11}=209 \mathrm{GPa}, \mathrm{C}_{12}=133 \mathrm{GPa}, \mathrm{C}_{44}=121 \mathrm{GPa}$ [27]. Franciosi et al. [28] obtained the interaction coefficients between stainless steel slip systems by hardening experiments, which could be chosen as $a_{1}=0.625$, $a_{2}=0.045, a_{3}=0.045, a_{4}=0.137, a_{5}=0.122$. The remaining unknown parameters could be determined by comparing the simulation results of RVE uniaxial tension with the measured stress-strain curves.

The average grain size and grain orientation information needed for simulation were obtained by the electron backscatter diffraction (EBSD) tests of the ultra-thin 304 stainless steel foil. EBSD diagrams of 304 stainless steel foil samples measured from the rolling direction (RD) and transverse direction (TD) are shown in Figure 2. The average grain size measured was $15 \mu \mathrm{m}$. The maximum density pole of the $\{111\}$ pole figure was only 1.97 m.r.d (multiple random density), which indicates that the annealed 304 foil had little texture and the grain orientations were almost random. Therefore, the random grain orientations were applied to the polycrystalline model based on the Voronoi polyhedron theory.
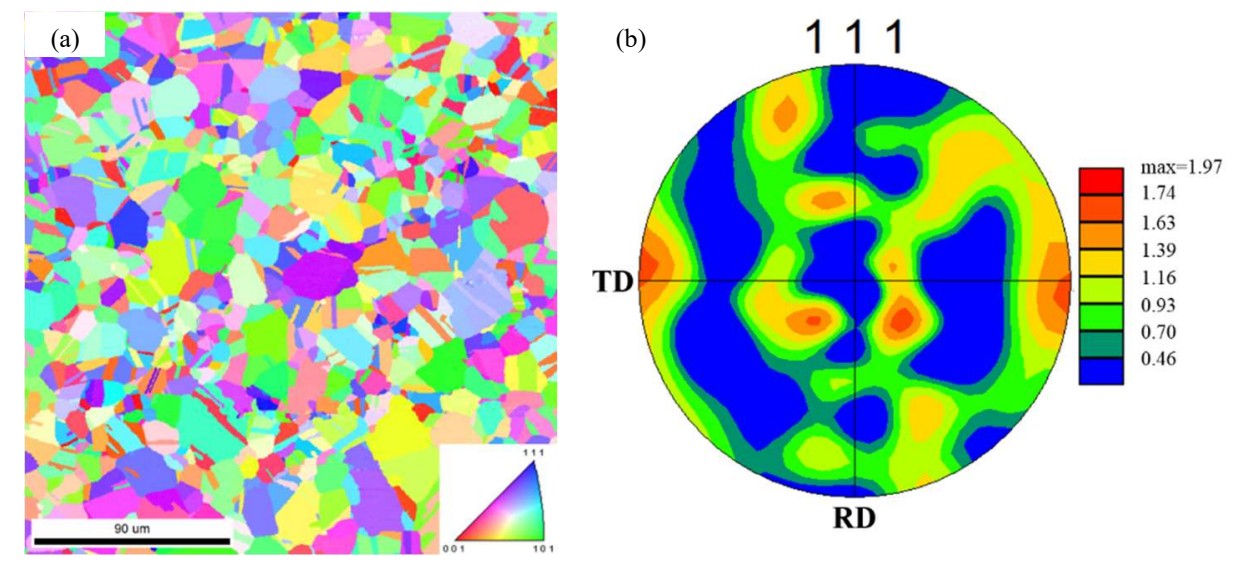

Figure 2. Electron backscatter diffraction (EBSD) mapping of the transverse direction (TD) measured from the rolling direction (RD) of 304 stainless steel foil: (a) grain orientation map; (b) $\{111\}$ pole figure.

The RVE model was established based on ABAQUS Standard. The effects of the number of grains and the number of elements in the grains on the tensile deformation accuracy of RVE model were analyzed, as shown in Figure 3. The RVE model contained 512 grains with a grain size of about $15 \mu \mathrm{m}$, and each grain contained 125 hexahedral elements (C3D8I), with average element dimensions of $3 \times 3$ $\times 3 \mu^{3}$ (Figure $4 \mathrm{~b}$ ), which could guarantee the calculation accuracy. Different colors in the model represent different grain orientations, and the aggregates of the same orientation are one grain.

(a)

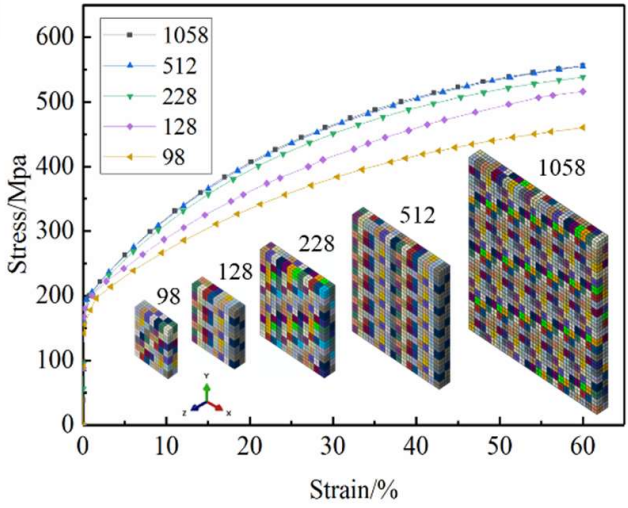

(b)

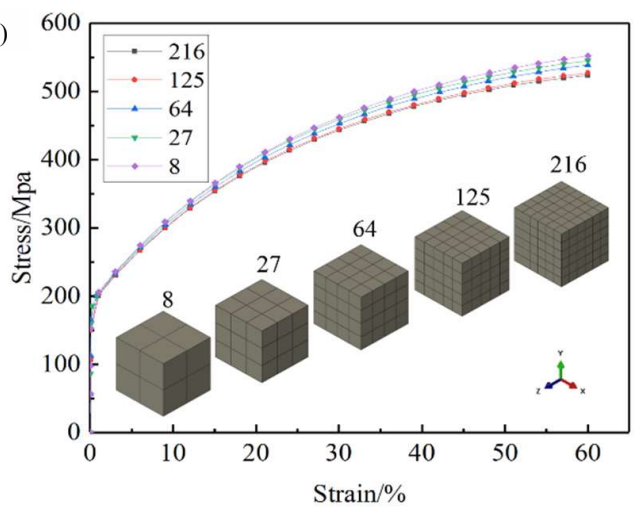

Figure 3. Effect of grain number (a) and unit number (b) on the representative volume element (RVE) model.

The boundary conditions of the RVE uniaxial tension model are shown in Figure 4a: 
(1) The back surface center node was set at $X=0, Y=0, Z=0$ to avoid the rigid motion of the RVE model as a whole.

(2) The back surface was set at $X=0$. There was no constraint in the $Y$ and $Z$ directions, so that plastic deformation could occur in the $\mathrm{Y}$ and $\mathrm{Z}$ directions.

(3) The front surface was set in a uniform tensile displacement along in the $X$-direction.

All calculations were run on a workstation ( $2.6 \mathrm{GHz}$ processor) with 20 multiple processors in parallel. The maximum number of increments was 100,000, to ensure convergence of the RVE model. It took around $5 \mathrm{~h}$ to complete one tension test of the RVE with $60 \%$ engineering strain.

According to the international standard ASTM-E345-16 [29], $0.05 \mathrm{~mm}$ thick 304 stainless steel foil tensile specimens were prepared and stretched using an INSTRON-5969 universal tensile testing machine (Instron corporation, Norwood, MA, USA). In the experiment, the stretching rate was $0.03 \mathrm{~mm} / \mathrm{min}$. The RVE model established above was subjected to uniaxial tensile simulation to an engineering strain of $60 \%$. The obtained stress-strain curves were compared with the curves measured by tensile tests. The reasonable crystal plastic parameters were determined by optimization (see Table 1), and the comparison results shown in Figure 5 were obtained.
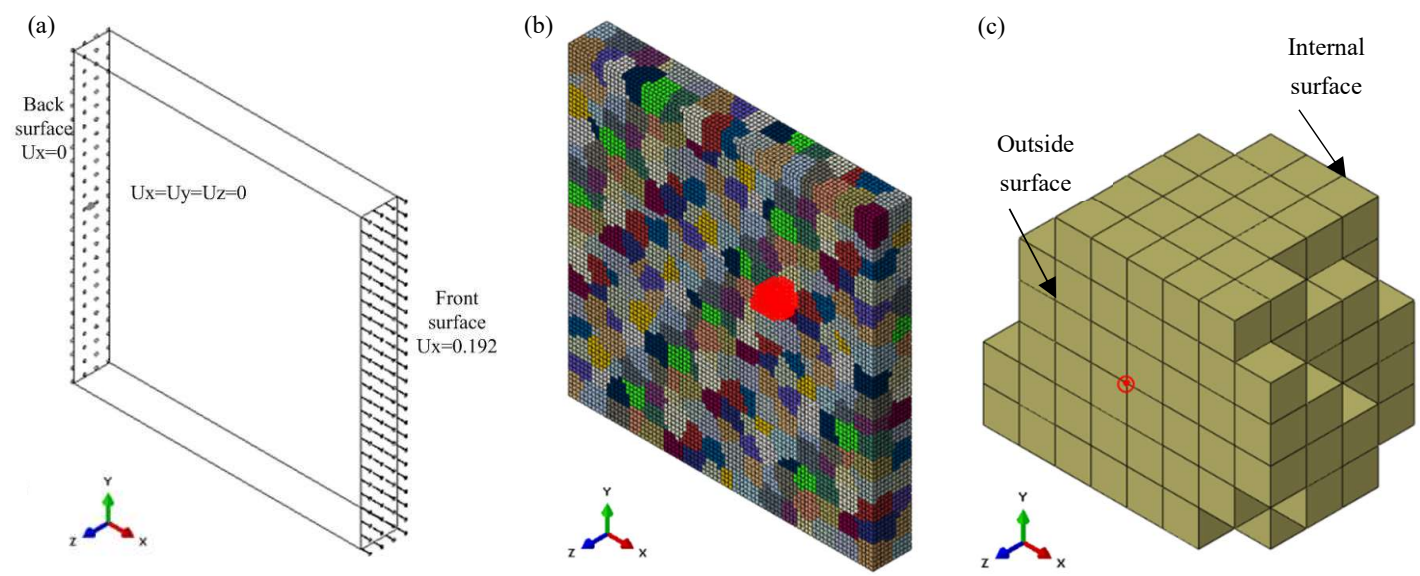

Figure 4. 304 stainless steel RVE tensile model: (a) boundary conditions in the simulation; (b) RVE model; (c) G411.

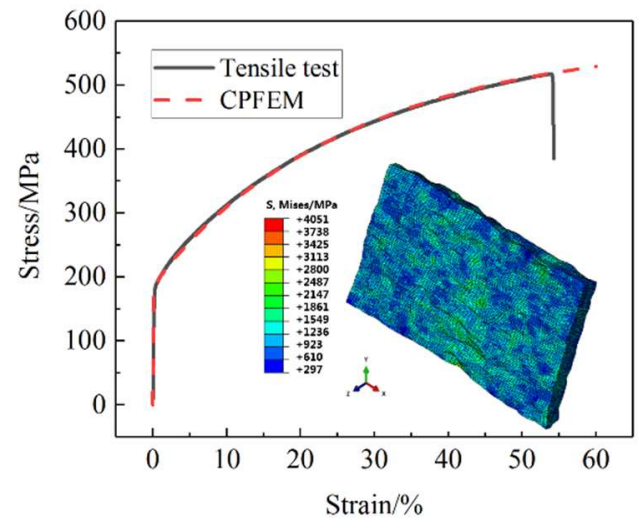

Figure 5. Comparison of the stress-strain curves between RVE simulation and experimental results.

Table 1. Constitutive model parameters of ultra-thin 304 stainless steel foil.

\begin{tabular}{cccccccc}
\hline $\mathbf{n}$ & $\dot{\gamma}$ & $\mathbf{h}_{0}$ & $\boldsymbol{\tau}_{1}$ & $\boldsymbol{\tau}_{0}$ & $\mathbf{h}_{\boldsymbol{s}}$ & $\boldsymbol{\gamma}_{0}$ & $\mathbf{q}$ \\
\hline 60 & 0.0001 & 245 & 30 & 74 & 105 & 0.001 & 1.0 \\
\hline
\end{tabular}


To study the effect of grain orientation on the motion of slip systems, five typical grain orientations (see Table 2) were assigned to the 411 grain (G411, Figure 4c) in the RVE model, and the other grains were given a random orientation, as shown in Figure 6. Then, the stress-strain curves of uniaxial tension to $60 \%$ engineering strain were obtained, as shown in Figure 7 . It is clear that the five stress-strain curves coincide completely, indicating that a change of single grain orientation does not affect the mechanical properties of the stainless steel ultra-thin foil. The accuracy of the RVE model established in this paper is verified.

The 304 stainless steel had an FCC crystal structure. The dislocation slip motion occurred on the 12 slip systems $\{111\}<110>$, as shown in Table 3. The [100] direction of the crystal was consistent with the X-axis, with [010] and [001] corresponding to the Y-axis and Z-axis, respectively. According to the theory of crystal plasticity, the initiation of the slip system is always controlled by the shear strain rate on the slip system. The negative value of shear strain rate indicates that the slip should be along the opposite direction to the slip system [30].

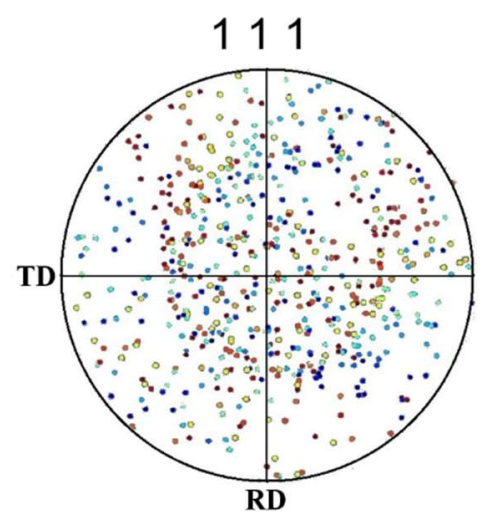

Figure 6. $\{111\}$ pole figure before RVE deformation with random orientation.

Table 2. Five typical grain orientations of G411.

\begin{tabular}{|c|c|c|c|c|}
\hline Number & Grain Orientation & Euler Angles & Miller Indices & Angle to X-Direction \\
\hline No.1 & Brass & $\left(35^{\circ}, 45^{\circ}, 90^{\circ}\right)$ & $\left(\begin{array}{lll}1 & 0 & 1\end{array}\right)\left[\begin{array}{ll}\overline{1} & \overline{2} \\
1\end{array}\right]$ & $110.56^{\circ}$ \\
\hline No.2 & Copper & $\left(90^{\circ}, 35^{\circ}, 45^{\circ}\right)$ & (1 112$)\left[\begin{array}{lll}\overline{1} & \overline{1} & 1\end{array}\right]$ & $125.26^{\circ}$ \\
\hline No.3 & $\mathrm{S}$ & $\left(61^{\circ}, 34^{\circ}, 64^{\circ}\right)$ & 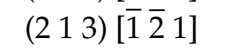 & $116.09^{\circ}$ \\
\hline No.4 & Cube & $\left(0^{\circ}, 0^{\circ}, 0^{\circ}\right)$ & $\left(\begin{array}{lll}0 & 0 & 1\end{array}\right)\left[\begin{array}{lll}1 & 0 & 0\end{array}\right]$ & $0^{\circ}$ \\
\hline No.5 & Goss & $\left(0^{\circ}, 45^{\circ}, 90^{\circ}\right)$ & $\left(\begin{array}{lll}1 & 0 & 1\end{array}\right)\left[\begin{array}{lll}0 & \overline{1} & 0\end{array}\right]$ & $90^{\circ}$ \\
\hline
\end{tabular}

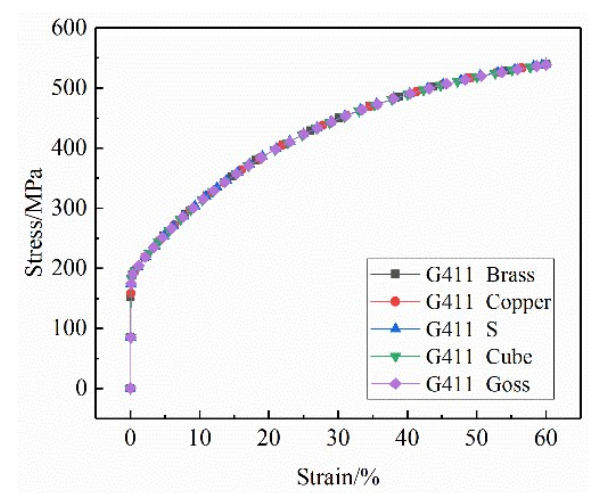

Figure 7. Stress-strain curves of G411 in the RVE model under five different grain orientations. 
Table 3. Slip systems of face-centered cubic FCC metal.

\begin{tabular}{|c|c|c|c|c|c|}
\hline Slip Plane & Slip Direction & Slip System & Slip Plane & Slip Direction & Slip System \\
\hline \multirow{3}{*}{$\left(\begin{array}{lll}1 & 1 & 1\end{array}\right)$} & 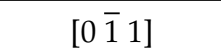 & a1 & \multirow{3}{*}{$(1 \overline{1} 1)$} & {$\left[\begin{array}{lll}0 & 1 & 1\end{array}\right]$} & c1 \\
\hline & {$\left[\begin{array}{lll}1 & 0 & \overline{1}\end{array}\right]$} & $\mathrm{a} 2$ & & {$\left[\begin{array}{lll}1 & 1 & 0\end{array}\right]$} & c2 \\
\hline & {$\left[\begin{array}{lll}\overline{1} & 1 & 0\end{array}\right]$} & a3 & & {$\left[\begin{array}{lll}1 & 0 & \overline{1}\end{array}\right]$} & c3 \\
\hline \multirow{3}{*}{$(\overline{1} 11)$} & {$\left[\begin{array}{lll}1 & 0 & 1\end{array}\right]$} & b1 & \multirow{3}{*}{$\left(\begin{array}{lll}1 & 1 & 1\end{array}\right)$} & {$\left[\begin{array}{lll}0 & 1 & 1\end{array}\right]$} & $\mathrm{d} 1$ \\
\hline & {$\left[\begin{array}{lll}1 & 1 & 0\end{array}\right]$} & b2 & & {$\left[\begin{array}{lll}1 & 0 & 1\end{array}\right]$} & $\mathrm{d} 2$ \\
\hline & {$\left[\begin{array}{llll}0 & \overline{1} & 1\end{array}\right]$} & b3 & & {$\left[\begin{array}{lll}1 & 1 & 0\end{array}\right]$} & $\mathrm{d} 3$ \\
\hline
\end{tabular}

\section{Results and Discussion}

\subsection{Microscopic Stress and Strain Distribution of G411}

Figure 8 shows the stress distribution of G411 under five typical grain orientations for the RVE models with $60 \%$ tensile engineering strain. It can be seen that the Brass, Copper, and S oriented grains had an obvious rotation phenomenon. The stress at the internal grain boundary was highly concentrated and the maximum stress value was high, even up to $2507 \mathrm{MPa}$ for the Copper-oriented grain. The grain morphologies of the Cube and Goss oriented grains were similar after tensile deformation, which was mainly elongated along the tensile direction. The stress distribution was relatively uniform and the maximum stress value was small. The maximum stress value of the Cube oriented grain was $2004 \mathrm{MPa}$. It can be concluded that the different initial grain orientations led to the different degrees of the grain deformation under the same deformation conditions, and the microscopic stress distribution and the maximum stress value differed greatly.

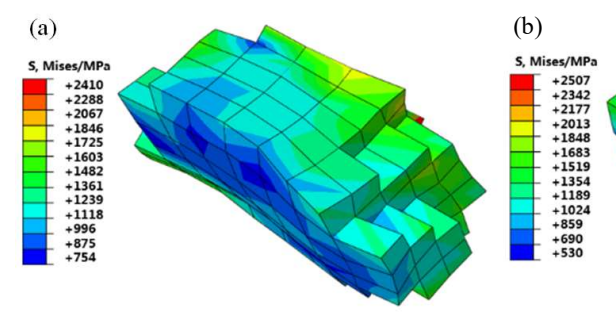

(d)

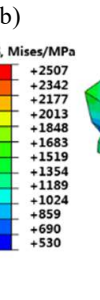

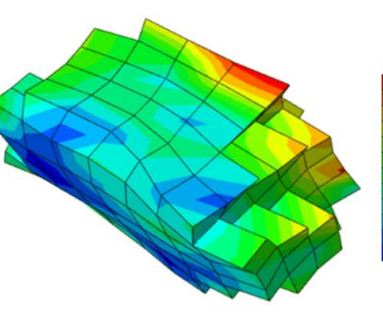
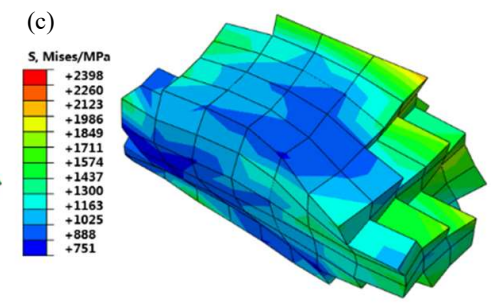

(e)
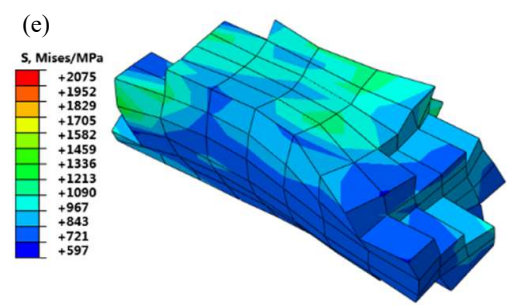

Figure 8. Grain morphology and Mises stress distribution after the G411 deformation: (a) Brass; (b) Copper; (c) S; (d) Cube; (e) Goss.

Figure 9 shows the accumulative slip distribution of G411 under five typical grain orientations for the RVE models with $60 \%$ tensile engineering strain. As can be seen, the cumulative shear strain distributions of five typical oriented grains were not uniform, due to the restraint of adjacent grains, and there were obvious strain gradient characteristics. The strain was mainly concentrated in the boundary region with the internal grain contact. The Cube and Goss oriented grains had large deformations along the $\mathrm{X}$-axis tensile direction. The cumulative shear strain distribution appeared to be more uniform and the cumulative shear strain value was small. The maximum cumulative shear strain value of the Cube oriented grain was 1.999 . It can be seen that the initial grain orientation directly affected the cumulative shear strain distribution of the grains.

Considering the deformation coordination of polycrystalline, the grain deformation can be affected by the constraints of adjacent grains. Due to the different initial grain orientations, the order of grain 
deformation varies with the starting sequence of slip systems in different parts of the grain, so the deformation degree of the grain is different [31]. Compared with the resistance overcome by the slip of the grain internal dislocation, the slip at the grain boundary needs to overcome the resistance caused by the coordination of intergranular deformation, so the stress of polycrystalline plastic deformation is concentrated at the grain boundary [32].

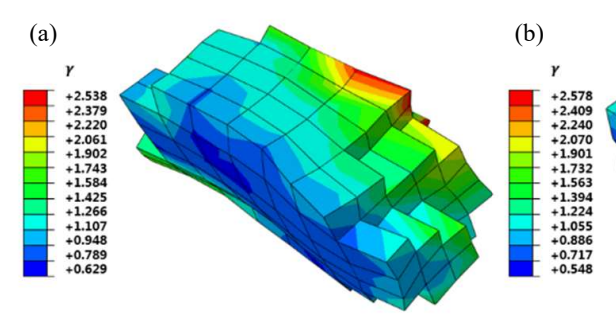

(d)

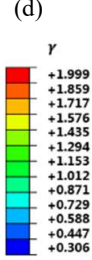

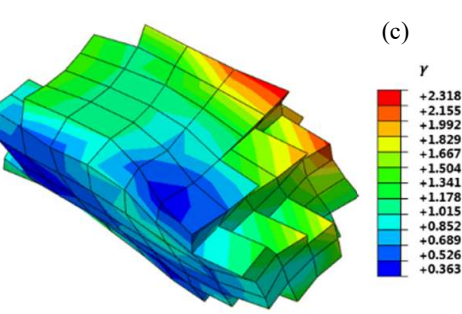

(e)

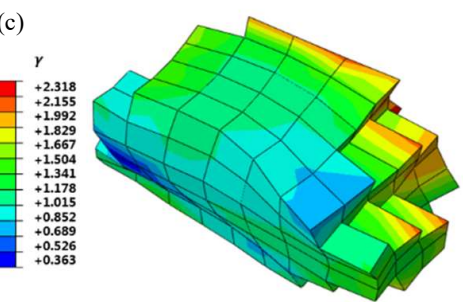

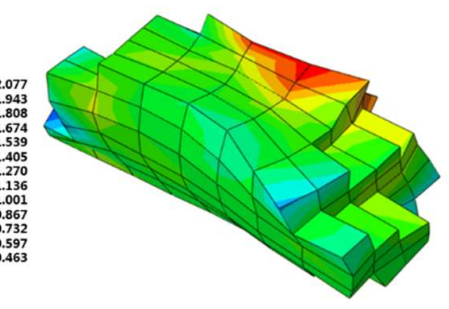

Figure 9. Cumulative shear strain distribution after the G411 deformation: (a) Brass; (b) Copper; (c) S; (d) Cube; (e) Goss.

\subsection{Slip System Evolution of G411 with the Typical Grain Orientations}

In order to investigate the relationship between grain orientation and shear strain rate of the slip systems during tensile deformation, a node on the outside surface of G411, shown in Figure 4c, was selected for analysis, and the results are shown in Figure 10. When the G411 grain orientation was Brass, Copper, or S-type, the number and motion state of the activated slip systems at the node were similar, because of the approximate angles between the three given orientations and the tensile direction. The slip systems a 2 and c 3 were activated in the above three orientations. Cube and Goss oriented grains had the largest number of activated the slip systems-eight. For the Cube oriented grain, the shear strain rates of slip systems a2, c2, c3, and d2 were large and slipped along their positive directions. The shear strain rates of slip systems a3, b1, b2, and d3 were small and slipped along their opposite directions. For the Goss oriented grain, the slip systems a1, b3, c1, and c2 slid along their opposite directions and the shear strain rates were large, while the slip systems a3, b2, d1, and d3 slid along their positive directions and the shear strain rates were small. It can be concluded that the number and motion state of the activated slip systems at the same position varied greatly because of the different initial grain orientations. The Cube and Goss oriented grains had the largest number of activated the slip systems in the uniaxial tensile stress state, which was beneficial to the occurrence of dislocation slip movement. 

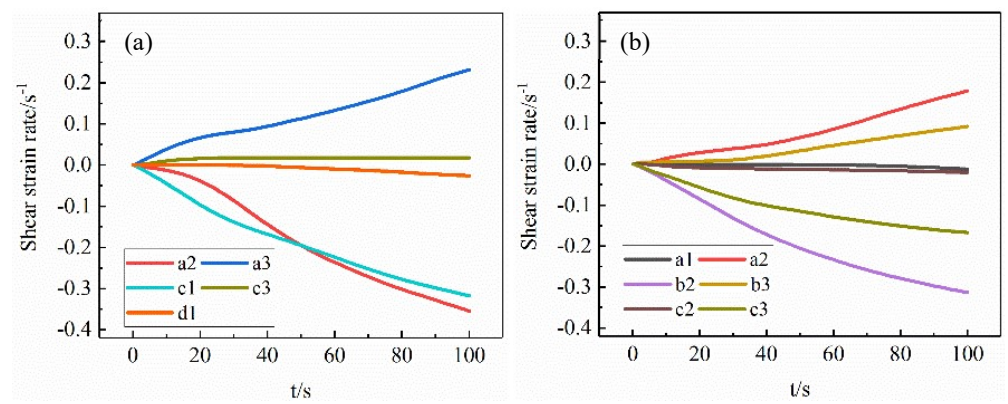

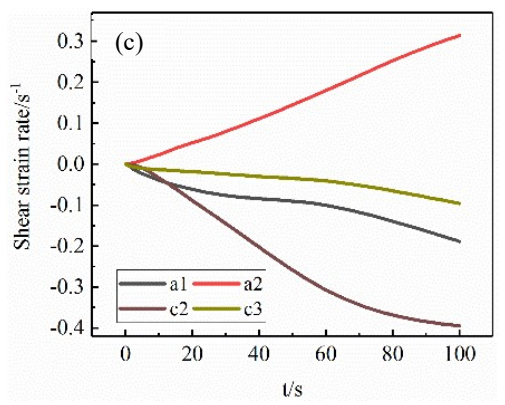

$1 / \mathrm{s}$
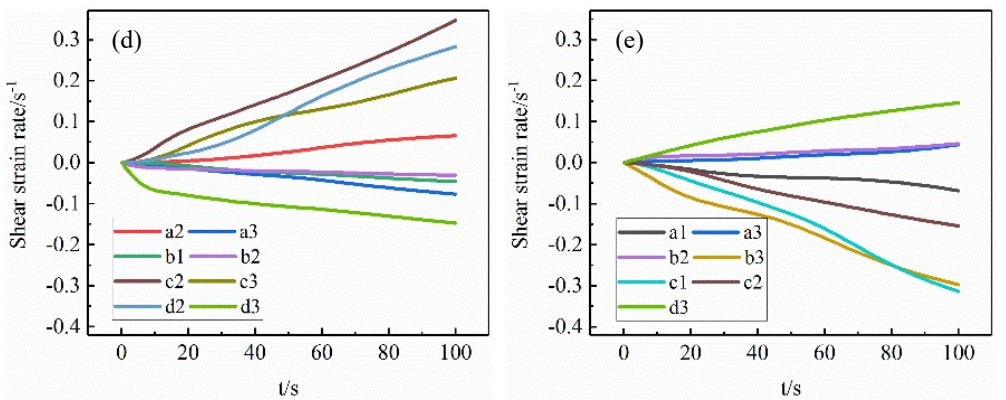

Figure 10. Shear strain rates of a node on the G411: (a) Brass; (b) Copper; (c) S; (d) Cube; (e) Goss.

The starting process of the 12 slip systems of FCC metal during uniaxial tension deformation is shown in Figure 11. When the grain orientation was Cube type, the local coordinate system of the crystal coincided with the global coordinate system of the sample and the angle between grain orientation and tensile force direction was zero. It can be seen that at this time the slip systems of $\mathrm{a} 1, \mathrm{~b} 3, \mathrm{c} 1$ and $\mathrm{d} 1$ were perpendicular to the direction of the force. Thus, shear strain did not occur and the shear strain rate was zero. For the Goss grain orientation, the crystal coordinate system did not coincide with the sample coordinate system and the angle between grain orientation and tensile force direction was $90^{\circ}$. At this time, the slip systems a2, b2, c3, d2 were perpendicular to the tensile direction and the shear strain rate was zero. As shown in Figure 10d,e, the other eight slip systems were activated except for the above four slip systems perpendicular to the tensile stress. The shear strain rates of each slip system are different due to the influence of grain morphology and adjacent grains. In summary, the movement of the slip systems in the process of tensile deformation was analyzed by simulation and theory, and the accuracy of the crystal plasticity finite element model was verified.
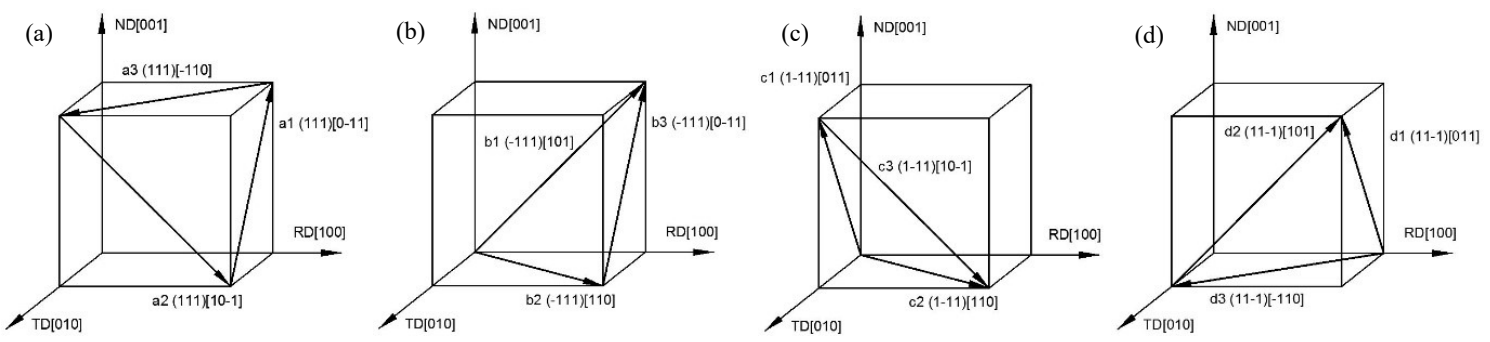

Figure 11. Illustration of $\{111\}$ planes for initial tensile process: (a) (111) slip plane; (b) (-111) slip plane; (c) (1-11) slip plane; (d) (11-1) slip plane.

Figure 12 shows the evolution of the shear strain rate of the slip systems a2 and c3 during uniaxial tensile deformation of the Brass oriented G411. It can be observed that the shear strain rate distribution of the slip systems was inhomogeneous. The slip appea red in the opposite direction inside the single crystal grain, which is conducive to the formation of sub-crystallines. The shear strain rate distribution of different slip systems was also various. The a2 and c3 slip systems were activated first at the grain boundaries. With the rotation of the slip surface and direction of the tensile deformation, the internal and free surface slip systems started. The slip strain rate distribution of the slip system a2 varied 
greatly from -0.321 to 1.956 . The outside surface nodes mainly moved along the opposite direction of the slip system a2. The difference of the shear strain rate of the slip system $\mathrm{c} 3$ in the whole grain was only 1.662, and the distribution was relatively uniform. It can be seen that the slip systems were activated at the grain boundary first and then caused the movement of the internal and free surface slip systems, and finally formed a slip band in the whole grain. At the same time, the above conclusions can be drawn from the analysis of the shear strain rate evolution process of the other four typical grain orientation slip systems.

Affected by grain orientation, morphology, and polycrystalline deformation compatibility, the number of the activated slip systems and the magnitude of shear strain at different locations are quite different. The motion state of the activated slip systems during plastic deformation is also different. These differences lead to inhomogeneous deformation of the grain and contribute to the stress-strain concentration zone [33].

(a) a2-1

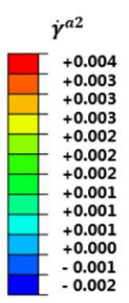

(d) $\mathrm{c} 3-1$

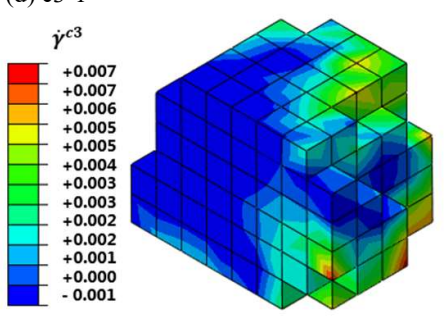

(b) a2-2

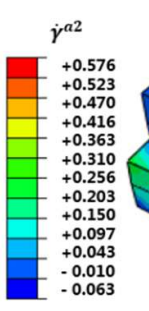

(e) $\mathrm{c} 3-2$

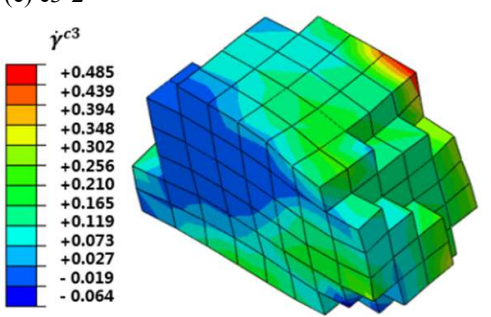

(c) a2-3

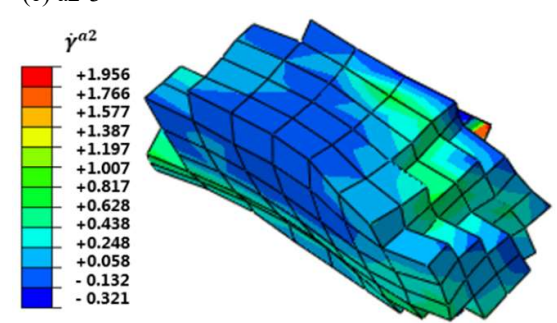

(f) $\mathrm{c} 3-3$

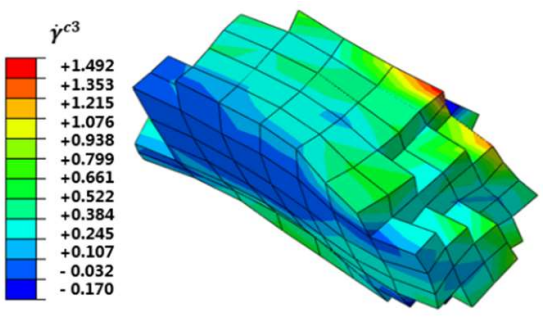

Figure 12. Shear strain rate along active slip system a2 (a2-1, a2-2, a2-3) and c3 (c3-1, c3-2, c3-3) for the G411 of Brass with the tensile time $0.5 \mathrm{~s}$ (a2-1, c3-1), $30 \mathrm{~s}$ (a2-2, c3-2), $100 \mathrm{~s}$ (a2-3, c3-3).

\subsection{Stress and Strain Curves of Typical Texture Orientation Dominant Polycrystalline}

An RVE model with a typical texture orientation was established. The number of grains with each dominant orientation accounted for $50 \%$ of the total grains. The simulated stress-strain curves are shown in Figure 13. As can be seen, the yield strength of the RVE model with the dominant texture orientation was approximately $193 \mathrm{MPa}$, but its deformation resistance varied greatly. In the plastic deformation stage, the stress-strain curves of Brass, S, and Copper oriented dominant models were higher than those of random grain oriented models. The stress-strain curves of Cube oriented and Goss oriented RVE models coincide basically and are significantly lower than those of random oriented RVE models. Under the $60 \%$ strain condition, the stress values of the Brass, S, and Copper oriented dominant models are higher than those of random grain oriented model. The maximum stress value of the Copper-type orientation-dominated model is $618 \mathrm{MPa}$. The stress values of Cube-type and Goss-type orientation-dominated models are approximately $495 \mathrm{MPa}$, which is lower than that of the random grain orientation model (538 MPa). The results show that Brass, $\mathrm{S}$, and Copper oriented stainless steel materials have large energy storage, large plastic deformation resistance, and a high strain hardening index, which are beneficial to strengthening. Cube-oriented and Goss-oriented stainless steels exhibit low strength and low deformation resistance, and are prone to plastic deformation. 


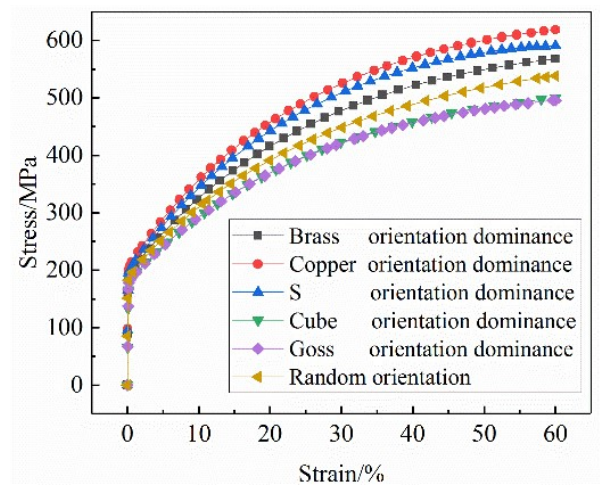

Figure 13. Comparison of stress and strain curves of RVE models with typical texture orientation.

\section{Conclusions}

(1) Based on the crystal plasticity theory and microscopic characterization analysis, a crystal plastic RVE model of 304 stainless steel foil was established. By comparing the tensile stress-strain curves between the RVE simulation and experiment, the calibrated crystalline plastic parameters can accurately reflect the mechanical properties of 304 stainless steel foil. Combined with the simulation and theoretical analysis of the evolution of slip systems during the tensile deformation process, the accuracy of the crystal plastic finite element model was verified.

(2) The crystal plasticity RVE model was used to simulate the effect of typical grain orientation on the inhomogeneous deformation of ultra-thin 304 stainless steel foil during tension. The results show that the numbers of start-up and movement state of the slip systems at the same location are quite different due to the various initial grain orientations, resulting in a great difference in grain deformation degrees and stress-strain distribution. The effect of grain orientation on the plastic deformation of polycrystals is mainly manifested in the mutual constraints and coordination of various grain deformation processes. During the tensile deformation process, the stress distribution and the shear stress with different slip systems are different due to the different initial grain orientation. Therefore, the initiating sequence of the slip systems is different, resulting in different deformation orders and deformation degrees. The grains in favorable orientations slip first, and the grains in unfavorable orientations are difficult to slip.

(3) The change of stress-strain curves in the tensile process of 304 stainless steel foil RVE model with typical orientation was analyzed by CPFEM. The results show that the Brass, $\mathrm{S}$, and Copper oriented dominant models exhibit a high strain hardening index and are beneficial to metal strengthening. However, Cube and Goss oriented dominant models have lower strength and low deformation resistance, so 304 stainless steel foil with these orientations are prone to plastic deformation.

Author Contributions: Z.R. conceived and guided the simulation analysis; W.F. wrote the paper; J.H. provided experiment data, materials and experimental equipments; T.W. revised the paper.

Funding: This research was funded by the National Natural Science Foundation of China (Grant No. 51804215), the China Postdoctoral Science Foundation (Grant No. 2019M651074), the Shanxi Province Science and Technology Major Projects (Grant No. 20181102015, 20181101008), the Taiyuan City Science and Technology Major Project (Grant No. 170203) and the Natural Science Foundation of Shanxi Province (Grant No. 201801D221221).

Conflicts of Interest: The authors declare no conflict of interest.

\section{References}

1. Xiao, H.; Ren, Z.; Liu, X. New mechanism describing the limiting producible thickness in ultra-thin strip rolling. Int. J. Mech. Sci. 2017, 133, 788-793. [CrossRef]

2. Chen, Z.J.; Chen, X.; Zhou, T.P. Microstructure and mechanical properties of J55ERW steel pipe processed by on-line spray water cooling. Metals 2017, 7, 150. [CrossRef] 
3. Daming, N.; Zhen, L.; Kaifeng, Z. Grain size effect of commercial pure titanium foils on mechanical properties, fracture behaviors and constitutive models. J. Mater. Eng. Perform. 2017, 26, 1283-1292. [CrossRef]

4. Fu, M.W.; Chan, W.L. Geometry and grain size effects on the fracture behavior of sheet metal in microscale plastic deformation. Mater. Des. 2011, 32, 4738-4746. [CrossRef]

5. Grogan, J.A.; Leen, S.B.; McHugh, P.E. Influence of statistical size effects on the plastic deformation of coronary stents. J. Mech. Behav. Biomed. Mater. 2013, 20, 61-76. [CrossRef] [PubMed]

6. Furushima, T.; Tsunezaki, H.; Manabe, K.I.; Alexsandrov, S. Ductile fracture and free surface roughening behaviors of pure copper foils for micro/meso-scale forming. Int. J. Mach. Tools Manuf. 2014, 76, 34-48. [CrossRef]

7. Chen, S.D.; Liu, X.H.; Liu, L.Z. Symmetric and asymmetric rolling pure copper foil: crystal plasticity finite element simulation and experiments. Acta Metall. Sin. (Engl. Lett.) 2015, 25, 1024-1033. [CrossRef]

8. Ye, W.; Efthymiadis, P.; Pinna, C.; Ma, A.; Shollock, B.; Dashwood, R. Experimental and modelling study of fatigue crack initiation in an Aluminium beam with a hole under 4-point bending. Int. J. Solids Struct. 2018, 138, 87-96. [CrossRef]

9. Ma, X.; Zhao, J.; Du, W.; Zhang, X.; Jiang, L.; Jiang, Z. An analysis of ridging of ferritic stainless steel 430. Mater. Sci. Eng. A 2017, 685, 358-366. [CrossRef]

10. Bishoyi, B.; Debta, M.K.; Yadav, S.K.; Sabat, R.K.; Sahoo, S.K. Simulation of texture evolution during uniaxial deformation of commercially pure Titanium. IOP Conf. Ser. Mater. Sci. Eng. 2018, 338, 012038. [CrossRef]

11. Singh, J.; Kim, M.S.; Choi, S.H. The effect of initial texture on micromechanical deformation behaviors in $\mathrm{Mg}$ alloys under a mini-V-bending test. Int. J. Plast. 2019, 117, 33-57. [CrossRef]

12. Si, L.Y.; Lü, C.; Tieu, A.K.; Liu, X.H. Simulation of polycrystalline aluminum tensile test with crystal plasticity finite element method. Trans. Nonferrous Metals Soc. China 2007, 17, 1412-1416. [CrossRef]

13. Hama, T.; Takuda, H. Crystal-plasticity finite-element analysis of inelastic behavior during unloading in a magnesium alloy sheet. Int. J. Plast. 2011, 27, 1072-1092. [CrossRef]

14. Hama, T.; Takuda, H. Crystal plasticity finite-element simulation of work-hardening behavior in a magnesium alloy sheet under biaxial tension. Comp. Mater. Sci. 2012, 51, 156-164. [CrossRef]

15. Ritz, H.; Dawson, P.R. Sensitivity to grain discretization of the simulated crystal stress distributions in FCC polycrystals. Modell. Simul. in Mater. Sci. Eng. 2009, 17, 015001. [CrossRef]

16. Pi, H.C.; Han, J.T.; Zhang, C.G.; Tieu, A.K.; Jiang, Z.Y. Modeling uniaxial tensile deformation of polycrystalline Al using CPFEM. J. Univ. Sci. Technol. Beijing 2008, 15, 43-47. [CrossRef]

17. Nakamachi, E.; Tam, N.N.; Morimoto, H. Multi-scale finite element analyses of sheet metals by using SEM-EBSD measured crystallographic RVE models. Int. J. Plast. 2007, 23, 450-489. [CrossRef]

18. Jigh, H.G.J.; Frsi, M.A.; Hosseini, T.H. Pre-diction of the stress-strain behavior of open-cell Aluminum foam under compressive loading and the effects of various RVE boundary conditions. J. Mater. Eng. Perform. 2018, 7, 2576-2585. [CrossRef]

19. Zhang, L.; Jing, W.G.; Wu, Z.H. Finite element simulation on mechanical properties of hierarchical honeycombs. J. Nan-chang Hangkong Univ. (Nat. Sci.) 2016, 30, 22-27.

20. Kamiński, M.; Sokołowski, D. Dual probabilistic homogenization of the rubber-based composite with random 373 carbon black particle reinforcement. Comp. Struct. 2016, 140, 783-797. [CrossRef]

21. Rehrl, C.; Völker, B.; Kleber, S.; Antretter, T.; Pippan, R. Crystal orientation changes: A comparison between a crystal plasticity finite element study and experimental results. Acta Mater. 2003, 51, 2379-2386. [CrossRef]

22. Smallman, R.E. Plasticity of crystals with special reference to metals by E. Schmid and W. Boas. J. Appl. Crystallogr. 2014, 25, 587-588.

23. Hutchinson, J.W. Bounds and self-consistent estimates for creep of polycrystalline materials. Proc. R. Soc. London A Math. Phys. Sci. 1976, 348, 101-127. [CrossRef]

24. Wu, T.Y.; Bassani, J.L.; Laird, C. Latent hardening in single crystals I. theory and experiments. Proc. R. Soc. London A Math. Phys. Sci. 1991, 435, 1-19. [CrossRef]

25. Bassani, J.L.; Wu, T.Y. Latent hardening in single crystals II. analytical characterization and predictions. Proc. R. Soc. London A Math. Phys. Sci. 1991, 435, 21-41. [CrossRef]

26. Kysar, J.W. Addendum to a User-Material Subroutine Incorporating Single Crystal Plasticity in the ABAQUS Finite Element Program; Mech report 178; Division of Engineering and Applied Sciences, Harvard University: Cambridge, MA, USA, 1997. 
27. Simmons, G.; Wang, H. Single Crystal Elastic Constants and Calculated Polycrystal Properties; MIT Press: Cambridge, UK, 1971.

28. Franciosi, P.; Berveiller, M.; Zaoui, A. Latent hardening in FCC crystals. In Strength of Metals and Alloys; Haasen, P., Gerold, V., Kostorz, G., Eds.; Elsevier Ltd.: Amsterdam, The Netherlands, 1979; Volume 1, pp. 23-28.

29. Methods for Tension Testing of Metallic Foil; ASTM E345-08; American Society of Testing and Materials: West Conshohocken, PA, USA, 2016.

30. Pan, W.K.; Wang, Z.Q.; Zhang, Y.W. Discrete slip model of crystal and finite element analysis. Acta mech. Sin. 1997, 29, 278-286.

31. Zhang, C.; Zhang, L.W.; Shen, W.F.; Xia, Y.N.; Yan, Y.T. 3 d crystal plasticity finite element modeling of the tensile deformation of polycrystalline ferritic stainless steel. Acta Metall. Sin. (Engl. Lett.) 2017, 30, 79-88. [CrossRef]

32. Si, L.Y. Simulation of the Texture Evolution During Cold Deformation of FCC Metal with Crystal Plasticity FEM. Ph.D. Thesis, Northeastern University, Shengyang, China, June 2009.

33. Chen, S.D.; Liu, X.H.; Liu, L.Z.; Song, M. Crystal plasticity finite element simulation of slip and deformation in ultra-thin Copper strip rolling. Acta Metall. Sin. 2016, 52, 120-128.

(C) 2019 by the authors. Licensee MDPI, Basel, Switzerland. This article is an open access article distributed under the terms and conditions of the Creative Commons Attribution (CC BY) license (http://creativecommons.org/licenses/by/4.0/). 